\title{
Normalizer of parabolic aubgroups in unitary reflection groups
}

\author{
KRISHNASAMY MURALEEdaran
}

\section{INTRODUCTION}

Let $G$ be a unitary reflection group acting on a vector space $V$ and $P$ be a parabolic subgroup of $G$, that is a pointwise stabilizer of a subspace of $V$. Then there is a subgroup $H$ of $G$ such that $N_{G}(P)=P H$ and $H \cap P=\{1\}$. In the process of proving this result, we find the structure of the normalizer of $G$ in the unitary group $U(V)$ of $V$ and generalize the result of Cameron, Goethals, Seidel and Shult [2] on star-closed line systems from the real to the complex case.

The concept of a unitary ( known as pseudo or complex ) reflection was introduced by Shephard [13] in the mid $20^{\text {th }}$ century and eventually Shephard and Todd [] published a list of all finite irreducible unitary (complex) reflection groups up to conjugacy. In this paper, they separately studied the imprimitive groups and primitive groups. They introduced the infinite family of groups $G(m, p, n)$, where $m, p$ and $n$ are positive integers with $p$ divides $m$, to study the imprimitive unitary reflection groups. The primitive unitary reflection groups were known by their corresponding collineation groups generated by homologies which were studied by G. Bagnera (1905), H.F. Blichfildt and H.H. Mitchell (1914). In 1967, Coxeter [5], in 1976, Cohen [3] and [4] and in 1990, Hughes [8] studied associated graphs whose vertices correspond to generators of irreducible unitary reflection groups. In the real case these graphs reduce to the usual Dynkin diagrams.

In 1974, Springer [15] studied these groups by introducing regular elements in them. (A vector is regular if it lies in no reflecting hyperplane; a group element is regular if it has a regular eigenvector.) Since then these groups have been studied extensively by many mathematician; notably G.I. Lehrer, P. Orlik, L. Solomon, M. Broué, G. Malle, R. Rouquier and K. Bremke.

Recent work of Broué, Malle and Michel [1] and the related work of Lehrer and Springer [9] have shown that irreducible unitary reflection groups are a crucial part of the study of algebraic groups.

Received 16th October, 2006

Thesis submitted to the University of Sydney, January 2005. Degree approved, April 2006. Supervisor: Associate Professor Don Taylor.

Copyright Clearance Centre, Inc. Serial-fee code: 0004-9727/07 \$A2.00+0.00. 


\section{Context of the thesis}

In this thesis we extend the work of Howlett [6], where he studied the structure of normalizers of parabolic subgroups in Coxeter groups, to unitary reflection groups. Even though unitary reflection groups have root systems they don't have the notion of positive roots (see [7] for detail). Hence there is no proper definition of length function nor of reduced word, which were the important tools in Howlett's calculations. Therefore, we study the structure of the normalizers of parabolic subgroups of unitary reflection groups case by case. Another recent study of the parabolic subgroups of imprimitive unitary reflection groups $G(m, 1, n)$ can be found in the paper of Mak [10].

In this process, we omit the case when a irreducible unitary reflection group is a Coxeter group. We calculate the normalizers separately for the cases when the irreducible unitary reflection group is imprimitive and primitive. For the imprimitive case, we use Orlik-Soloman's theory (see [12]) to classify all the parabolic subgroups and calculate their normalizers (see Chapters 4 and 5 of the thesis). In the primitive case, we mainly use the index of normalizers of parabolic subgroups in irreducible unitary reflection groups which are given in [12] (see Chapters 6 and 7 of the thesis). The calculations in Chapters 6 and 7 were verified by MAGMA, the powerful software tool in algebra developed in University of Sydney. For this case, we also need to study the structure of normalizers of all irreducible unitary reflection groups in their respective unitary groups (see Chapter 3 of the thesis) and to study the complex root line systems of some irreducible unitary reflection groups (see Chapter 2 of the thesis). In the process of studying complex root line systems, we were able to prove a generalization of a result of Cameron, Goethals, Seidel and Shult [2] and classify all the primitive unitary reflection groups of rank greater than 4. In this context, we use the Theorems of Mitchell [11]. By studying the structures of normalizers of parabolic subgroups, in each case we find that the parabolic subgroup is complemented in its normalizer.

\section{REFERENCES}

[1] M. Broué and J. Michel, 'Towards spetses. I', Transform. Groups 4 (1999), 157-219.

[2] P.J. Cameron, J.M. Boethals, J.J. Seidel and E.E. Shult, 'Line graph, root system and elliptic geometry', J. Algebra 43 (1076), 305-327.

[3] A.M. Cohen, 'Finite complex reflection groups', Ann. Sci. École Norm. Sup. (4) 9 (1976), 379-436.

[4] A.M. Cohen, 'Erraftum: Finite complex reflection groups', Ann. Sci. École Norm. Sup. (4) 11 (1978), 613 .

[5] H.S.M. Coxeter, 'Finite groups generated by unitary reflections', Abh. Math. Sem. Univ. Hamburg 31 (1967), 125-135.

[6] R.B. Howlett, 'Normalizers of parabolic subgroups of reflection groups', J. London Math. Soc. (2) 21 (1980), 62-80. 
[7] R.B. Howlett and J.-Y. Shi, 'On regularity of finite reflection groups', Manuscripta Math. 102 (2000), 325-333.

[8] M.C. Hughes, 'Complex reflection groups', Comm. Algebra 18 (1990), 3999-4029.

[9] B.I. Lehrer and T.A. Springer, 'Reflection subquotients of unitary reflection groups', Canad. J. Math. 51 (1999), 1175-1193.

[10] C.K. Mak, 'Quasi-paraboic subroups of $g(m, 1, r)$ ', J. Algebra 246 (2001), 471-490.

[11] H.H. Mitchell, 'Determination of all primitive collineation groups in more than four variables which contain homologies', Amer. J. Math. 36 (1914), 1-12.

[12] P. Orlik and L. Solomon, 'Arrangements defined by unitary reflection groups', Math. Ann. 261 (1982), 339-357.

[13] G.C. Shephard, 'Unitary groups generatted by reflections', Canad. J. Math. 5 (1953), 364-383.

[14] G.C. Shephard and J.A. Todd, 'Finite unitary reflection groups', Canad. J. Math. 6 (1954), 274-304.

[15] T.A. Springer, 'Regular elements of reflection groups', Invent. Math. 25 (1974), 159-198.

Department of Maths UGRU

United Arab Emirates University

Al-Ain

United Arab Emirates

e-mail: muraleek@uaeu.ac.ae 